\title{
Biologically Inspired Architecture of Feedforward Networks for Signal Classification
}

\author{
Šarūnas Raudys and Minija Tamošiūnaitè \\ Institute of Mathematics and Informatics \\ Vytautas Magnus University \\ Vileikos 8, Kaunas LT-3035, Lithuania \\ raudys@das.mii.lt, minija.tamosiunaite@vdu.lt
}

\begin{abstract}
The hypothesis is that in the lowest hidden layers of biological systems "local subnetworks" are smoothing an input signal. The smoothing accuracy may serve as a feature to feed the subsequent layers of the pattern classification network. The present paper suggests a multistage supervised and "unsupervised" training approach for design and training of multilayer feed-forward networks. Following to the methodology used in the statistical pattern recognition systems we split functionally the decision making process into two stages. In an initial stage, we smooth the input signal in a number of different ways and, in the second stage, we use the smoothing accuracy as a new feature to perform a final classification.
\end{abstract}

\section{Introduction}

Large number of hidden layers is the essential feature of modern pattern classification systems based on neural networks $[4,8,10]$. Most popular technique in a multilayer perceptron (MLP) classifier training is a gradient descent based back propagation (BP) algorithm. However, the algorithm suffers from long learning times required to obtain a solution. More complex algorithms such as conjugent gradient, or quasi Newton methods, are faster, however, are more sensitive to local minima problems [9]. Two typical operations performed in each MLP neuron are a weighted summation and a nonlinear transformation. While training a MLP the error signal propagates from an output layer back to hidden layers and is used to update the weights of the network. A change in the weights is proportional to the error signal and to the gradient of the cost function calculated over a number of neurones in the upper layers of the network. With an increase in the number of iterations MLP weights are increasing and lead to diminution of the gradients. Thus, irrespective to a learning set (empirical) error, the training process slows down. This problem is especially noticeable in the feed-forward networks with a high number of hidden layers. In complex real world pattern classification problems, however, the high number of hidden layers is a typical situation. As a result, the number of hidden layers and the non-linear character of the activation function constitute main reasons of the slow training speed. 
Propagation of the error signal for a great number of layers is not probable while training biological neural networks. We suppose that the weight change in such systems is performed by a simpler mechanism, and hypothesize that in lower hidden layers of biological systems "local subnetworks" are smoothing input signals. The smoothing accuracy may serve as a feature to feed the subsequent layers. In this paper we suggest a multistage supervised and "unsupervised" training approach for design and training of complex multilayer feed-forward networks. We smooth the input signals in a number of different ways and use the smoothing accuracy as a new feature supplied to the next network's layer. This approach has much in common with traditional techniques in pattern recognition, where input layers are utilized for feature extraction.

Our argumentation, however, is rather different: we do not use artificial mathematical methods (spectral, cepstral features, coefficients of autoregression or moving average model coefficients, one or two dimensional Gabor, Fourier filters, see e.g. [5]) for the feature extraction. Instead, we suggest to train the input layers of the network to smooth the signal and to calculate the smoothing accuracy. We use selected typical signals to train the hidden layer subnetworks for signal prediction. Later in the course of the algorithm prediction accuracy is used to form the new features. The number of the selected typical signals determine the number of features utilized in the final recognition stage of the decision making network. In order to explain the main idea, below we present the utilization of our multistage supervised and unsupervised training algorithm to solve a problem of classification of electrocardiografic signals. To make our explanation as simple as possible we analyze a relatively simple network, consisting of several hidden layers and one output layer. The first hidden layer performs signal smoothing. This layer is not trained - it is defined apriori. The upper hidden layers are trained to predict the signal from its several adjacent values and to estimate the prediction accuracy. The output layer performs the final classification and is trained by the conventional BP technique.

\section{ECG Classification Problem}

The ECG classification is vital in determining susceptibility of an individual to sudden cardiac death. Analysis of an electrocardiogram (ECG) up to the last decade has been grounded on examination of low frequency information (P,Q,R,S,T waveforms; position and length of some intermediate segments). Higher frequency information, which is usually less in energy, was excluded from analysis because of the relatively low accuracy of available recordings. The increase in accuracy of recordings, as well as in computer power, has encouraged seeking for information on the state of heart among the higher frequency oscillations (e.g. presence of late potentials in ST segment have been discovered and their prognostic value proven [2]). The current study is focused on examination of the high frequency oscillations found on a $T$ wave, which by itself has attracted enormous interest of cardiologists during the last few years. Possibility of the high frequency component analysis is provided by 

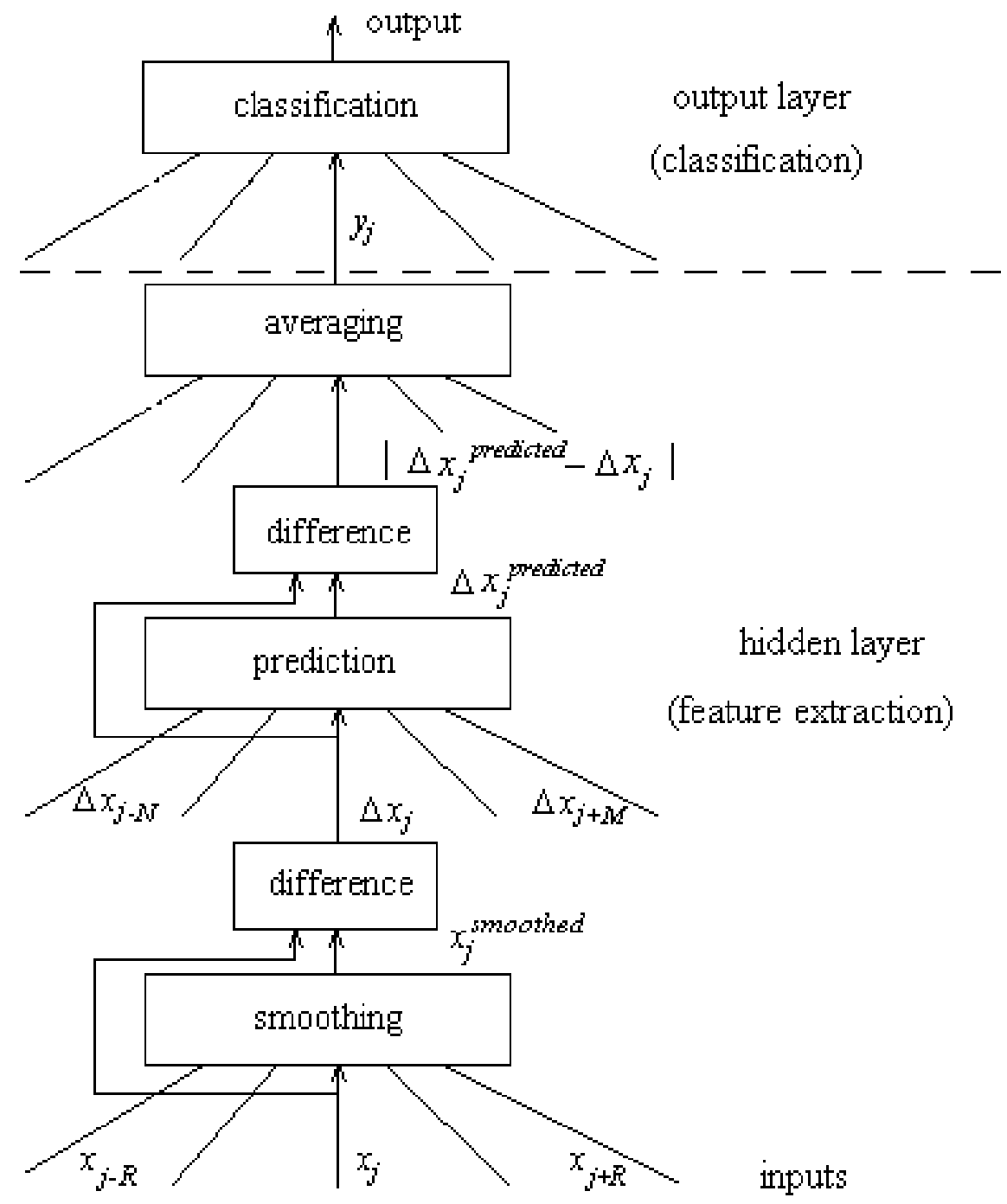

Fig. 1. The training schema of the multilayer network for signal classification

high accuracy of the available ECG recordings ( 12 bits, $2 \mathrm{kHz}$ discretization). Intrinstic properties of the high frequency oscillations are considered to be represented by autoregression models. Neural networks are supposed to discover informative components, which normally are mixed with noise arising during the ECG recording.

The two classes to be delineated by the proposed method are: myocardial infarction (MI) patients who have had the complication of ventricular fibrillation (VF), as opposed to those whose MI was not complicated by the VF. The task of distinguishing risk of life threatening ventricular fibrillation is important, but no reliable solutions have been achieved as yet [11]. 


\section{The ANN Training Strategy and the Architecture of Network}

The proposed network consists of the hidden layer and the output layer. The sequence of layers is selected to resemble feature extraction procedures performed in living systems. The specific characteristic of the network is its step by step training algorithm introduced instead of the overall BP training procedure.

The hidden layer. Here, in the first step, smoothing of the original signal $X=\left\{x_{1}, x_{2}, \ldots, x_{n}\right\}$ is performed:

$$
x_{j}^{\text {smoothed }}=\sum_{i=-R}^{R} v_{i} x_{j-i},
$$

where $v_{-R}, v_{-R+1}, v_{R+2}, \ldots, v_{R-1}, v_{R}$ are values of a smoothing window, such that $\sum_{i=-R}^{R} v_{i}=1$, and $R$ represents the window width; $i=1,2, \ldots, n$. The values of parameters $\boldsymbol{v}=\left\{v_{i}\right\}$ which form the weights of the first network layer are chosen task-specific, no training is done.

In the second step, the high frequency signal $\Delta X=\left\{\Delta x_{1}, \Delta x_{2}, \ldots, \Delta x_{n}\right\}$ is extracted from the original signal, using the smoothed one:

$$
\Delta x_{j}=x_{j}-x_{j}{ }^{\text {smoothed }}, j=1,2, \ldots, n .
$$

No training is required in this step.

In the third step, a set $S=\left\{S_{1}, S_{2}, \ldots, S_{p}\right\}$ of "typical" high frequency signals from both pattern classes is selected, and the corresponding set of linear neural networks is trained for the signal prediction:

$$
\Delta x_{j}^{\text {predicted }}=\sum_{i=-N}^{i=M} w_{j \neq 0} \Delta x_{i+j},
$$

where $w_{i}{ }^{k}$ is the $i$-th weight of the $k$-th prediction rule, $j=1,2, \ldots, n, k=1,2, \ldots, p$. Parameters $\boldsymbol{w}=\left\{w_{i}{ }^{k}\right\}$ form the weights of the second hidden layer. In the classification phase, $p$ units perform prediction of an input sequence. As a result, we have $p$ sequences $\Delta x_{j}^{k \text {,predicted }}, \quad j=1,2, \ldots, n-N-M, \quad k=1, \ldots, p$. Together, all three steps perform a weighted summation.

In the fourth step, "similarities" $y_{1}, y_{2}, \ldots, y_{p}$ of a sequence to be classified $\Delta X=\left\{\Delta x_{1}, \Delta x_{2}, \ldots, \Delta x_{n}\right\}$ to the typical signals $S_{1}, S_{2}, \ldots, S_{p}$ are calculated:

$$
y_{k}=f\left(\Delta x_{i}-\Delta x_{i}^{k, \text { predicted }}, i=1,2, \ldots . n, z\right),
$$

where $\Delta x_{i}^{k, \text { predicted }}$ denotes $x_{i}$ predicted using the weight set $w_{j}^{k}, k=1,2, \ldots, p, \quad j$ $=-N,-N+1, \ldots,-1,1,2, \ldots, M ; z$ denotes a parameter set for the function $f$. The function $f$ includes averaging of the differences $\Delta x_{i}-\Delta x_{i}^{k \text {, smoothed }}$ in partially intersecting 


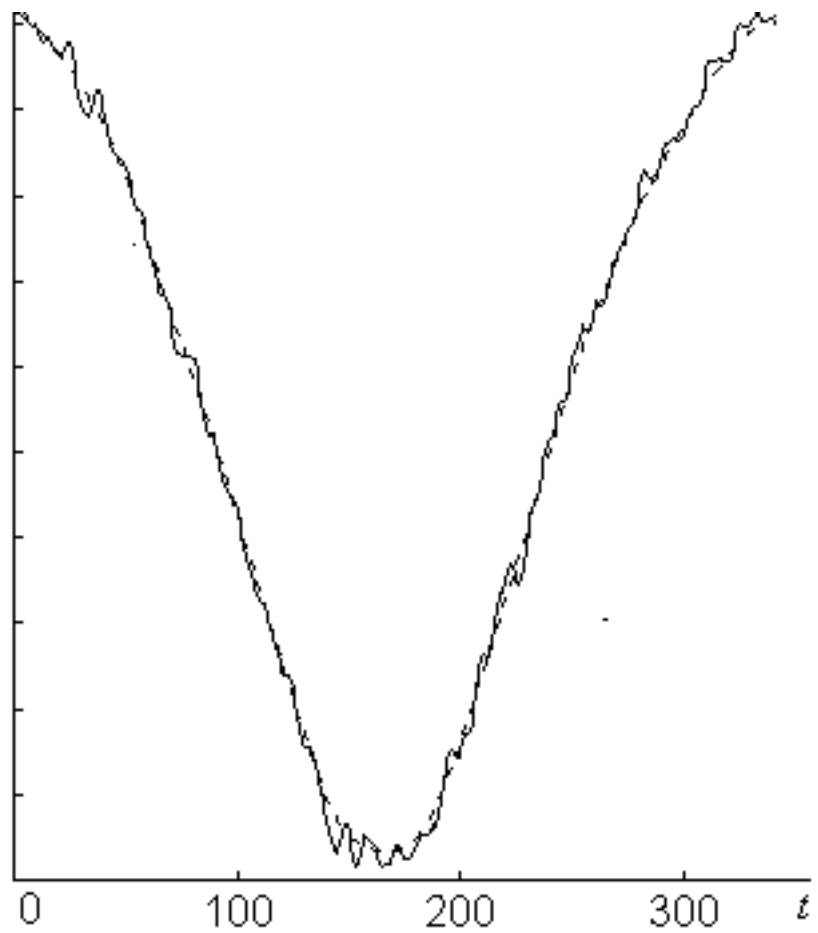

Fig. 2. The original $T$ wave (solid curve) and the smoothed signal (dotted curve) by means of triangular window with a basis $(-2020)$.

subintervals of the original signal and finding the median value of these averages. Parameters $z$ control the averaging process.

The information processing schema of all four steps is summarized in Fig. 1. Actually all four steps perform a weighted summation of the input signals and a nonlinear operation. In this sense, in a classification phase, an information processing procedure of all four steps can be realized by a non-linear single layer perceptron (SLP). The non-linear SLP also performs a weighted summation of input signals and a nonlinear operation [6].

The output layer is trained by conventional BP algorithm to assign the set of values $y_{1}, y_{2}, \ldots, y_{p}$ to the specific class. Entire recognition algorithm has been shown in Fig. 1. The information processing schema corresponds to that of one hidden layer MLP.

\section{Application of the Algorithm to ECG Analysis}

The last achievements in ECG analysis are dominated by features which are told not to be seen by a naked eye. This concerns the mentioned earlier high frequency components [16] and dynamical patterns arising in the long run of an ECG [3]. In 
analysis of the class of ECGs with ventricular fibrillation as opposed to those without $\mathrm{VF}$, high frequencies of the cardiosignals are guessed to contain significant part of information, that should be used for classification purposes. Therefore, it is worth analyzing the difference between the original and the smoothed signals

$\Delta x_{j}$ $=x_{j}-x_{j}^{\text {smoothed }}$ as given in the algorithm above.

In our ECG classification problem, the weights of the first layer of the network (eq. 1) were fixed apriori: we used a triangular window with $=R-|j|+1, j=0, \pm 1, \pm 2, \ldots, \pm R$. An example of the original and the smoothed signal of one particular $T$ wave is presented in Fig. 2.

To extract the useful information for classification, the network should calculate similarities $y_{1}, y_{2}, \ldots, y_{p}$ of the sequence $\Delta X$ to be classified, to $p$ typical $T$ waves selected from both classes. For this purpose in the training process of the overall neural network we design $p$ prediction rules, one for each of the selected $T$ waves.

In this particular pattern recognition task we have chosen to use a simple linear prediction rule (3). An example of the difference signal $\Delta X$ (solid curve) and the predicted signal $\Delta x_{j}^{\text {predicted }}$ (dotted curve) is presented in Fig. 3. The weights used for prediction are derived not from the signal itself, but from the other $T$ wave, selected as typical during the training procedure. It is evident that the difference between these two curves in the given example are minute, prompting the suggestion that the given signal could belong to the same class as the selected prototype.

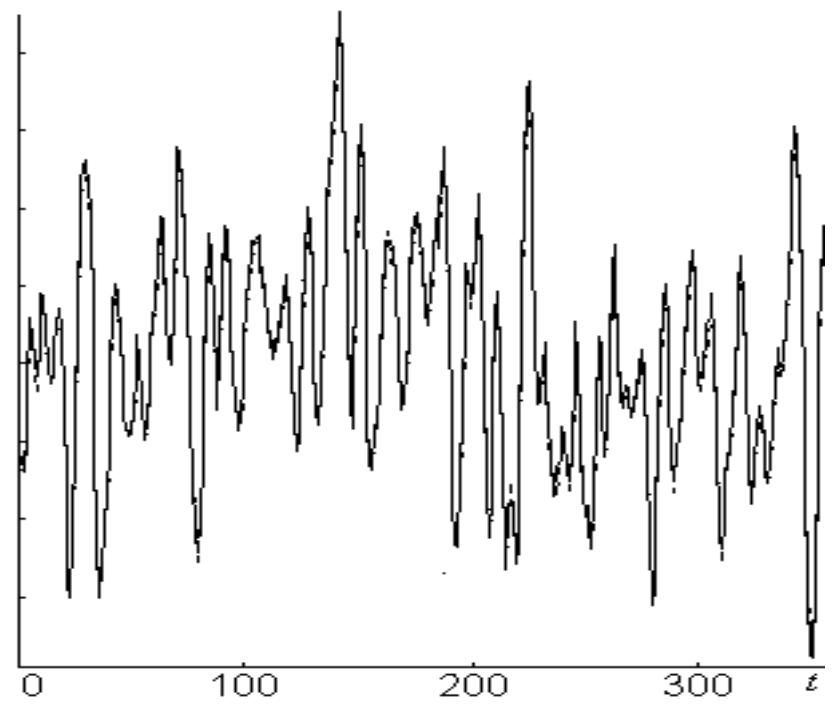

Fig. 3. The difference between the smoothed signal and its prediction by means of a linear equation $x_{t}=-0.2350 x_{t-2}+0.6849 x_{t-1}+0.8204 x_{t+1}-0.2663 x_{t+2}+1.7139$. 
In the classification phase, for a number (say $J$ ) of partially intersecting subintervals of the $T$ wave we calculated $J$ averages, of absolute values of deviations , $i=1,2, \ldots, L$ where $L$ is the length of the subinterval. Let us call the average over the subinterval. Mean values $y_{k}=\overline{\overline{y_{k}^{j}}}$ of the average prediction errors evaluated over all $J$ subintervals of the $T$ wave of the ECG signal were used as the new features. Above, index $k$ denotes a current number of the typical signal, $\quad k=1,2, \ldots, p$. In order to obtain robust estimates we utilized a sigmoid function to reduce contribution of largest $(\approx 10 \%)$ deviations. A sequence of new parameters $y_{1}, y_{2}, \ldots, y_{p}$ was used to make a final classification rule by means of a conventional single layer perceptron.

\section{Experiments and Results}

To verify the compound network's architecture, and the weights estimation schema, we used two category data set composed of $T$ waves obtained from 59 myocardial infarction (MI) patients who did not have the complication of ventricular fibrillation, and 43 ones who had suffered this problem. For training we used $29+22$ patients and for testing - the rest $29+21$ patients. After a couple of experiments with $R=20$ and $R=60$ for the signal smoothing we selected $R=20$. After the signal smoothing and finding the differences $\Delta x_{j}=x_{j}^{\text {smoothed }}-x_{j}$ in order to find the weights for each of the 51 individual $T$ waves we trained the linear single layer perceptron (3) to predict a "middle value" from $N+M$ values of the signal, and measured the prediction accuracy evaluated in this particular $T$ wave of the ECG signal. $23 T$ waves with the highest prediction accuracy were selected as "typical" $(p=23)$. The threshold for selection was:

$$
s^{\text {prediction }}<0.13 s\left(\Delta x_{j}\right)
$$

where $s\left(\Delta x_{j}\right)$ is a sample standard deviation of absolute values $\left|\Delta x_{j}^{\text {predicted }}-\Delta x_{j}\right|$, $j=1,2, \ldots, n$. Then the particular set of weights $\left\{w_{-N}^{k}, w_{-N+1}^{k}, w_{N+2}^{k}, \ldots, w_{-1}^{k}, w_{1}^{k}, \ldots, w_{M-1}^{k}\right.$, $\left.w^{k}{ }_{M}\right\}$ of prediction equation (3) was selected as parameters of the neurons of the second stage of the network. In our pattern classification problem, we tested $M=$ $N=1,2,3,4$ and 5 and found $M=2$ was enough to obtain sufficiently high prediction accuracy. We selected 14 "similarity" $T$ waves from the first pattern class and 9 from the other one. Thus, our network contained 23 hidden units that calculated "similarities", the new secondary features of the signal.

As the decision making schema in the output layer, we selected a simple single layer perceptron (SLP) trained by a back propagation algorithm. For training we used $5 T$ segments of each from $29+22$ patients investigated. For testing we also used $5 T$ segments of each from the rest $29+21$ patients. Thus, for training we used 25523 variate vectors, and for testing we had 250 vectors. 
To speed up the training process and to improve generalization we began the training from zero initial weight vector of the perceptron, used moving of a mean of the training data to a center of coordinates, rotated and scaled the data as it was suggested in $[14,15]$. In order to obtain the minimal classification error, we utilized an "antiregularization" technique described in [13]. To increase the magnitudes of the perceptron's weights and to force a cost function to minimize an empirical frequency of misclassifications we added to the standard sum of squares cost function a positive term $+0.1 \times\left(\boldsymbol{V}^{T} \boldsymbol{V}-100\right)^{2}$ and stopped training optimally (after 4100 batch iterations). $P_{\text {generalisation }}=P_{\text {test }}=0.304$ was achieved. A classification matrix of the test set is presented in Table 1 (left part).

Table 1. The classification matrices of the test set (left - without voting, right - with voting)

\begin{tabular}{l|rl}
\hline Pattern class & \multicolumn{1}{|c}{1} & 2 \\
\hline 1 (without VF) & 127 & 18 \\
2 (with VF) & 58 & 47 \\
\hline
\end{tabular}

\begin{tabular}{l|lc}
\hline Pattern class & 1 & 2 \\
\hline 1 (without VF) & 28 & 1 \\
2 (with VF) & 10 & 11 \\
\hline
\end{tabular}

As it was noted before, in our investigation, each patient was characterized by 5 ECG $T$ segments. Therefore, further improvement in the accuracy was obtained by a voting procedure: the generalization error was reduced down to $22 \%$ (Table 1, right). In comparison with conventional MLP training, the achieved accuracy is much higher: earlier, while classifying individual ECG $T$ segments we obtained 37-39\% of errors, just slightly better than a randomized classification according to the class prior probabilities, $P_{1}=0.58$ and $P_{2}=0.42$. In general, the achieved level of accuracy in sudden death recognition is reasonably high according to the standards of nowadays medicine.

\section{Concluding Remarks}

Above we presented the simple example that illustrates our approach to training of complex feedforward neural networks. Instead of training all layers of the network by the conventional BP technique we are trying to simplify the training process overloading a part of the work to the deterministic selection of weights and to unsupervised training. In the first layers of the proposed neural values of neighboring components of input vectors are predicted, while in the later stage, prediction accuracy is evaluated and final decision is made.

In the feature definition phase, we do not use the class membership indexes. Instead, we use the neighboring information from the process under investigation in order to determine the weights. Averaged errors of prediction of neighboring cells serve as features, supplied to the higher layers of the network. Some neurobiological evidence exists that such type of information processing is characteristic to "natural information processing systems" [12]. Moreover, the decorrelation of the inputs technique utilized while training the output layer of the network agrees with the results 
of investigations of visual cortex in biological systems. In an analysis of retinal ganglion cells, it was proposed that the purpose of retinal processing is to improve the efficiency of visual representation by recording the input into a spatially decorrelated form [1]. A bit later the decorrelation dynamics of lateral interaction between orientation selective cells in the early visual cortex was shown to yield quantitative predictions about orientation contrast, adaptation, and development of orientation selectivity which are in good agreement with experiments $[6,7]$.

Our analysis of the complex cardiology problem has shown that this simple method allowed to achieve rather high classification accuracy only on basis of ECG signal's $T$ wave measured for each patient. The achieved accuracy is considered high in the field of ECG analysis for sudden death prediction.

Our main goal was to illustrate that the very simple network can be used to solve rater complex real world problem. For this we need to decompose a global task into separate steps and perform training for each step separately. The solutions obtained are more stable, they do not require to make assumptions about mathematical models of the data. While training we have milder local minima problem, the number of weights to be learned is much smaller than while training the MLP classifier. Thus, from the theoretical point of view, such training strategy should require less training samples and should lead to better generalization properties.

Furthermore, the architecture of the network can be expanded. Instead of the simple triangular smoothing one can use more complex window function. Instead of the plain linear prediction in the feature definition stage, one can use complex MLP or Radial Basic Functions (RBF) networks. For final decision making one can also use MLP or RBF networks. The approach should be adapted specifically to each real world problem in question. It has been successfully used for identification of EEG states and financial times series prediction. In the latter case, instead of using SLP in input layers and output ones we were obliged to use MLP. The MLP classifiers in input layers were trained using different subsets of training data selected by a special neural network based algorithm and a financial analyst.

Acknowledgements -- The authors are thankful to Prof. Juozas Bluțas from Institute of Cardiology, Kaunas for providing the real-world data sets for the experiment and Dr. Erinija Pranckevičienë from Kaunas University of Technology for aid and stimulating discussions while working with EEG data.

\section{References}

1. Atick J.J., Redlich A.N.: Towards a theory of early visual processing. Neural Computation 2 (1990) 308-320.

2. Blužaitė I., Blužas, J., Brazdžionyte, J.: Signal-averaged electrocardiogram. Peculiarities of the first and recurrent Myocardial Infarction. J. HK Coll. Cardiol. 5 (1997) 119-25 
3. Burattini, L., Zareba, W. , Rashba, E.J. et al.: ECG Features of Microvolt T-Wave Alternans in Coronary Artery Disease and Long QT Syndrome Patients. Journal of Electrocardiology, 31 (1997) 114-120

4. LeCun, Y., Boser, B, Denker, J.S., Henderson, D.,.Howard, R.E., Hubbard, W., Jackel, L.D.: Backpropagation applied to handwritten zip code recognition, Neural Computation, 1 (1990) 541-551

5. Devijver P.A., Kittler J.: Pattern Recognition. A Statistical Approach, Precentice-Hall International, Inc., London (1982)

6. Dong, D.W.: (1993) Associative deccorelation dynamics in visual cortex, Lawrence Berkeley Laboratory Technical Report, LBL-34491 (1993)

7. Dong, D.W.: Associative deccorelation dynamics: a theory of self organization and optimization in feedback networks. In: Tesauro G, Touretzky DS, Leen TK (eds) Advances in neural information processing systems. MIT Press, Cambridge, MA, 7 (1994) 925-932

8. Fogelman-Soulie F., Vinnet E., Lamy B.: Multimodular neural network architectures: Application in optical character and human face recognition, Int. J. on Pattern recognition and Artificial Intelligence, 5 (1993) 721-755.

9. Gorse D., Shepherd, A., Taylor, J.G.: Avoiding local minima by a classical range expansion algorithm. In: I. Aleksander and J. Taylor (eds.) Artificial neural Networks, 2 , Elseviere (1992)

10. Haykin, S: Neural networks: A comprehensive foundation, Macmillan College Publishing Company, Inc., NY (1999)

11. Myerburg, R.J., , A., Mitrani, R.M.: Frequency of Sudden Cardiac Death and Profiles of Risk. American Journal of Cardiology 80 (5B, 1997) 10F-19F.

12. Quinlan, P.T.: Structural change and development in real and artificial neural networks. Neural Networks, 11 (1998) 577-599.

13. Raudys, S.: Evolution and generalization of a single neurone. I. SLP as seven statistical classifiers. Neural Networks 11 (1998) 283-296

14. Raudys, S.: Scaled rotation regularization. Pattern Recognition, 33 (2000, in press)

15. Raudys, S., Saudargiene, A.: Structures of the covariance matrices in the classifier design. Advances in Pattern Recognition. Springer Lecture notes in computer science. Vol. 1451 (Proc. joint IAPR int. workshops / SSPR'98 and SPR'98, August 11-13, 1998, Sydney, Australia) 583-592

16. Simson, M.B.: Use of signals in the terminal QRS complex to identify patients with ventricular tachycardia after myocardial infarction. Circulation 64 (1981) 235-242 